\title{
Azoniaallene salts as versatile building blocks in the synthesis of antibacterial and antifungal heterocyclic compounds
}

\author{
El-Sayed H. El-Tammany ${ }^{1}$, Atef A. Hamed ${ }^{2}$, Salah Z. A. Sowellim ${ }^{1}$, Ahmed S. Radwan $^{1 *}$ \\ ${ }^{1}$ Department of Chemistry, Faculty of Science, University of Suez Canal, Ismailia, Egypt; \\ *Corresponding Author: a radwan2000@yahoo.com \\ ${ }^{2}$ Department of Chemistry, Faculty of Science, Menofia University, Shebin El-Koom, Egypt
}

Received 10 October 2012; revised 12 November 2012; accepted 26 November 2012

\begin{abstract}
Substituted 2-azoniaallene salts 1 are strong bifunctional electrophiles, undergo cyclization reactions furnish many series of heterocyclic compounds, where reacted with $p$-tolyl urea, phenyl thiourea and thiosemicarbazone derivatives to afford triazinium salts, and converted to corresponding free bases 3, 5, 7 under treatment with $\mathrm{Na}_{2} \mathrm{CO}_{3}$. While triazole derivatives 8 and 9 were obtained by the reaction 2-azoniaallene salts 1 with benzohydrazide and phenyl hydrazine, respectively. Benzoxazinium salts 10 and 11 were acquired when asymmetric 2-azoniaallene salt reacted in (1:1) ratio with $p$-cresol and 3-methyl1-phenyl-5-pyrazolone, respectively. The reaction of 2-azoniaallene salt with malononitrile furnished the primidinium salt 12 which underwent neutralization with $\mathrm{Na}_{2} \mathrm{CO}_{3}$ followed by heterocyclization with hydrazine hydrate afforded the bicyclic compound 3-aminopyrazolo[3,4-d]pyrimidine 14 , which is highly reactive for nucleophilic addition to phenyl isothiocyanate to furnish thiourea derivative 15. Moreover, 14 undergo condensation with aldehydes to give imine derivatives $16 a, b$. All free base compounds were screened for their antimicrobial activities.
\end{abstract}

Keywords: Azoniaallene Salts; Triazines; Triazoles; Pyrazolo[3,4-d]primidines; Antimicrobial Activity

\section{INTRODUCTION}

During the last years methods have been developed for the synthesis of 2-azoniaallene salts, a class of heterocumulenes, which was discovered by Samuel and Wade [1, 2] in 1968. Salts of the general type $\mathbf{1}$ out to be valuable electrophiles for synthetic purposes. According to Ritter reaction, $\alpha$-chlorocarbenium ion reacts with nitriles to give initially an $\alpha$-chloronitrilium salts, which rearranges via a 1,3-chlorotropic shift to the thermodynamically more stable 2-azoniaallene salt [3], 2-azoniaallene also prepared by reaction of chlorocarbenium ion with 2,4,6-tris(trimethylsiloxy)-1,3,5-triazine to yield the 1oxa-3-azoniabutatriene salts, which react with ketones, carboxamides, or aldehydes affording 2-azoniaallene salts. A series of reactions take place to afford 2-azoniaallene salts, like reaction of diarylchlorocarbenium salts with potassium cyanate afforded the butatrienium salts which react with ketones to give 2-azoniaallene salts [4]. Ketones react with carbamyl chlorides in presence of antimony pentachloride afforded the corresponding azoniaallene salts. 1,3-Dichloro-2-azoniaallene salts without a stabilizing amino substituent [5] have only recently become available. Compounds $\mathbf{1}$ are strong bifunctional electrophiles reacting with heteronucleophiles [6], olefins, and acetylenes to give heterocycles and open-chain compounds. The theoretical studies on cycloaddition reactions of azoniaallene salts to produces five-member heterocycles were reported [7], 1,3-dipolar of cycloaddition reactions of neutral 1,3-dipoles have been developed into a generally practical method for fivemember heterocyclic-ring synthesis [8], and these reactions have been extensively studies both experimentally [9] and theoretically [10]. Benzisothiazol derivatives have been reported as cycloadditions products of azoniaallene salts with electron-rich alkenes [11]. Cycloaddition of azoniaallene salts to synthesis a variety of biologically active heterocyclic molecules [12], pyrazoles derivatives as antibiotics $[13,14]$, thiadiazole as antimycotic $[15,16]$ and anti-inflammatory agents $[17,18]$. Moreover triazole moiety is common in certain antiasthmatic [19], antivial [20] and hypnotics (triazolam) [21,22]. Synthesis of triazolium salts, thiadiazolium salts, C- and $\mathrm{N}$-nucleosides formed by reaction of azoniaallene salts with isothiocyanates have been reported $[23,24]$. The objective of our work is to generate new triazine, triazole, 
oxazine, and primidine derivatives based on the reaction of 2-azoniaallene salts with various reagents and study their antibacterial and antifungal activities.

\section{MATERIALS AND METHODS}

\subsection{Chemistry}

All solvents were dried by standard methods. All experiments for the synthesis of hexachloroantimonate salts were carried out with exclusion of moisture. The melting points were determined with Electrothermal (Barstead 9100) apparatus and are uncorrected. IR spectra were recorded with Shimadzu (IR Prestige-21) FT-IR spectrometer. ${ }^{1} \mathrm{H}$ and ${ }^{13} \mathrm{C}$ NMR spectra were determined with Jeol $\left({ }^{1} \mathrm{H}\right.$ NMR, ${ }^{13} \mathrm{C}$ NMR: $\left.400 \mathrm{MHz}\right)$, the chemical shifts in ppm are expressed on the $\delta$ scale using tetramethylsilane as internal standard. Mass spectra were measured with GC-MSQP 1000Ex Shimaduz. Microanalyses were performed with EuroEA Elemental Analyzer.

General procedure: Synthesis of 1,3-dichloro-1,3substituted-2-azoniaallene hexachloro-antimonates (1): A solution of $\mathrm{SbCl}_{5}(2.99 \mathrm{~g}, 10 \mathrm{mmol})$ in 1,2-dichloroethane $(10 \mathrm{~mL})$ was added dropwise at $-30^{\circ} \mathrm{C}$ to a well stirred solution of trichlorophenylmethane derivatives $(10 \mathrm{mmol})$ and nitrile derivatives $(10 \mathrm{mmol})$ in $40 \mathrm{~mL}$ 1,2-dichloroethane. When an orange precipitate was formed, the mixture was stirred at $-30^{\circ} \mathrm{C}$ for 15 minutes, heated to $+23^{\circ} \mathrm{C}$ within 10 minutes and boiled under reflux for 10 minutes. The reaction mixture was used for cycloaddition reaction without isolation.

4-Oxo-2,6-diphenyl-3-p-tolyl-3,4-dihydro-[1,3,5]triazin-1-ium hexachloroantimonate (2a): From trichloro phenylmethane $(1.96 \mathrm{~g}, 10 \mathrm{mmol})$ and benzonitrile $(1.03$ $\mathrm{g}, 10 \mathrm{mmol}$ ) as described for $\mathbf{1}$ and after cooling to $+23^{\circ} \mathrm{C}$, a suspension of $\mathrm{N}$-( $p$-tolyl)urea $(1.5 \mathrm{~g}, 10 \mathrm{mmol})$ in 1,2-dichloroethane was added to the mixture. The reaction mixture was boiled under reflux for 2 hours, and then cooled to room temperature. The solvent was removed under reduced pressure and the residue recrystallized from dichloroethane to afford orange fine crystal. Yield: $(5.1 \mathrm{~g}, 75.5 \%)$, m.p. $215^{\circ} \mathrm{C}-218^{\circ} \mathrm{C}$. IR $(\mathrm{KBr}): v=$ $1654(\mathrm{C}=\mathrm{N}), 1739(\mathrm{C}=\mathrm{O}), 3282 \mathrm{~cm}^{-1}(\mathrm{NH}) .{ }^{1} \mathrm{H}$ NMR (DMSO-d $\left.\mathrm{d}_{6}\right): \delta=2.26\left(\mathrm{~s}, 3 \mathrm{H}, \mathrm{CH}_{3}\right), 7.27-8.42(\mathrm{~m}, 14 \mathrm{H}$, Ar-H), 11.33 (s, 1H, NH). Anal. Calcd for $\mathrm{C}_{22} \mathrm{H}_{18} \mathrm{Cl}_{6} \mathrm{~N}_{3} \mathrm{OSb}$ (674.88): C, 39.15; H, 2.69; N, 6.23. Found: C, 39.38; H, $2.56 ; \mathrm{N}, 6.08$.

6-(Methylthio)-4-oxo-2-phenyl-3-p-tolyl-3,4-dihydr o-[1,3,5]triazin-1-ium hexachloroantimonate (2b): From trichlorophenylmethane $(1.96 \mathrm{~g}, 10 \mathrm{mmol})$ and methyl thiocyanate $(0.73 \mathrm{~g}, 10 \mathrm{mmol})$ as described for 1 and after cooling to $+23^{\circ} \mathrm{C}$, a suspension of $\mathrm{N}$ - $(p$-tolyl)urea $(1.5 \mathrm{~g}, 10$ $\mathrm{mmol}$ ) in 1,2-dichloroethane was added to the mixture. The reaction mixture was boiled under reflux for 2 hours, and then cooled to room temperature. The solvent was removed under reduced pressure and the residue recrystallized from dichloromethane/diethylether to afford yellow powder. Yield: $(3.5 \mathrm{~g}, 54.2 \%)$, m.p. $218^{\circ} \mathrm{C}-221^{\circ} \mathrm{C}$. IR (KBr): $v=1755(\mathrm{C}=\mathrm{O}), 3282 \mathrm{~cm}^{-1}(\mathrm{NH}) .{ }^{1} \mathrm{H}$ NMR $\left(\right.$ DMSO- $\left._{6}\right): \delta=2.40\left(\mathrm{~s}, 3 \mathrm{H}, \mathrm{CH}_{3}\right), 2.60\left(\mathrm{~s}, 3 \mathrm{H}, \mathrm{CH}_{3}\right)$, $7.53-8.57$ (m, 9H, Ar-H), 11.40 (s, 1H, NH). Anal. Calcd for $\mathrm{C}_{17} \mathrm{H}_{16} \mathrm{Cl}_{6} \mathrm{~N}_{3} \mathrm{OSSb}$ (644.87): C, 31.66; H, 2.50; N, 6.52. Found: C, 31.40; H, 2.35; N, 6.64 .

4,6-Diphenyl-1-p-tolyl-1H-[1,3,5]triazin-2-one (3): A mixture of the hexachloroantimate $2 \mathrm{a}(6.75 \mathrm{~g}, 10 \mathrm{mmol})$ in $\mathrm{CH}_{2} \mathrm{Cl}_{2}(15 \mathrm{~mL})$ and $\mathrm{Na}_{2} \mathrm{CO}_{3}(5.30 \mathrm{~g}, 50 \mathrm{mmol})$ in water $(30 \mathrm{~mL})$ was stirred for 3 hours. The organic layer was separated, the aqueous solution extracted with $\mathrm{CH}_{2} \mathrm{Cl}_{2}(2 \times 50 \mathrm{~mL})$ and the combined organic extracts were dried $\left(\mathrm{Na}_{2} \mathrm{SO}_{4}\right)$. The solvent was evaporated and the residue was crystallized from dichloromethane/diethyllether to afford yellowish orange crystals. Yield: $(2 \mathrm{~g}$, $58.9 \%)$, m.p. $173^{\circ} \mathrm{C}-176^{\circ} \mathrm{C}$. IR $(\mathrm{KBr}): v=1697(\mathrm{C}=\mathrm{O})$ $\mathrm{cm}^{-1}$. ${ }^{1} \mathrm{H}$ NMR: $\delta=2.26\left(\mathrm{~s}, 3 \mathrm{H}, \mathrm{CH}_{3}\right), 7.13-8.44(\mathrm{~m}$, $14 \mathrm{H}$, Ar-H). Anal. Calcd for $\mathrm{C}_{22} \mathrm{H}_{17} \mathrm{~N}_{3} \mathrm{O}$ (339.39): C, 77.86; H, 5.05; N, 12.38. Found: C, 77.62; H, 5.25; N, 12.18 .

2,6-Bis-(4-chlorophenyl)-3-phenyl-4-thioxo-3,4-dihydro-[1,3,5]triazin-1-ium hexachloroantimonate (4): From $p$-chlorotrichlorophenylmethane $(2.30 \mathrm{~g}, 10 \mathrm{mmol})$ and $p$-chlorobenzonitrile $(1.37 \mathrm{~g}, 10 \mathrm{mmol})$ as described for $\mathbf{1}$. After cooling to $+23^{\circ} \mathrm{C}$, a suspension of N-phenylthiourea $(1.52 \mathrm{~g}, 10 \mathrm{mmol})$ in 1,2-dichloroethane was added to the mixture. The reaction mixture was boiled under reflux for 3 hours, the solvent was removed under reduced pressure and the residue recrystallized from dichloromethane/diethylether to afford red fine crystal. Yield: $(6.0 \mathrm{~g}, 80.5 \%)$, m.p. $223^{\circ} \mathrm{C}-226^{\circ} \mathrm{C}$. IR $(\mathrm{KBr}): v=$ $3240 \mathrm{~cm}^{-1}(\mathrm{NH}) .{ }^{1} \mathrm{H}$ NMR (DMSO-d 6 ): $\delta=7.53-8.57$ (m, 13H, Ar-H), 10.82 (s, 1H, NH). Anal. Calcd for $\mathrm{C}_{21} \mathrm{H}_{14} \mathrm{Cl}_{8} \mathrm{~N}_{3} \mathrm{SSb}$ (745.80): C, 33.82; H, 1.89; N, 5.63. Found: C, 33.64; H, 2.02; N, 5.52.

4,6-Bis(4-chlorophenyl)-1-phenyl-1H-[1,3,5]triazine -2-thione (5): $\mathrm{A}$ mixture of the hexachloroantimonate salt $4(3.72 \mathrm{~g}, 5 \mathrm{mmol})$ in $\mathrm{CH}_{2} \mathrm{Cl}_{2}(15 \mathrm{~mL})$ and $\mathrm{Na}_{2} \mathrm{CO}_{3}$ $(5.30 \mathrm{~g}, 50 \mathrm{mmol})$ in water $(30 \mathrm{~mL})$ was stirred for 3 hours. The organic layer was separated, the aqueous solution extracted with $\mathrm{CH}_{2} \mathrm{Cl}_{2}(2 \times 50 \mathrm{~mL})$ and the combined organic extracts are dried $\left(\mathrm{Na}_{2} \mathrm{SO}_{4}\right)$. The solvent was evaporated and the residue was crystallized from hexane to afford violet powder. Yield: $(1.2 \mathrm{~g}, 58.8 \%)$, m.p. $180^{\circ} \mathrm{C}-183^{\circ} \mathrm{C}$. IR $(\mathrm{KBr}): v=1520,1585,3092$ $\mathrm{cm}^{-1} .{ }^{1} \mathrm{H}$ NMR (DMSO-d $\left.\mathrm{d}_{6}\right): \delta=7.53-8.57(\mathrm{~m}, 13 \mathrm{H}$, $\mathrm{Ar}-\mathrm{H}) . \mathrm{MS} \mathrm{m} / \mathrm{z}=411$ (22.3\%), 214 (32.1), 155 (100\%), 77 (62.1\%). Anal. Calcd for $\mathrm{C}_{21} \mathrm{H}_{13} \mathrm{Cl}_{2} \mathrm{~N}_{3} \mathrm{~S}$ (410.32): C, 61.47; H, 3.19; N, 10.24. Found: C, 61.19; H, 3.34; N, 10.02 .

2,4-Diphenyl-thiazolo[3,2-a][1,3,5]triazin-5-yliumhexachloroantimonate (6a): From trichlorophenylme- 
thane $(1.96 \mathrm{~g}, 10 \mathrm{mmol})$ and benzonitrile $(1.03 \mathrm{~g}, 10$ mmol) as described for 1 . After cooling to $+23^{\circ} \mathrm{C}$, Ntrimethylsilyl-aminothiazol [25] (1.72 g, $10 \mathrm{mmol})$ in absolute dichloroethane was added to the mixture. The reaction mixture was boiled under reflux for 3 hours. After cooling to room temperature, the solvent was removed under reduced pressure and the residue recrystallized from dichloroethane to afford yellowish white powder. Yield: $(5.3 \mathrm{~g}, 84.8 \%)$, m.p. $210^{\circ} \mathrm{C}-213^{\circ} \mathrm{C}$. IR $(\mathrm{KBr}): v=1539,1566,1581,3116 \mathrm{~cm}^{-1} .{ }^{1} \mathrm{H}$ NMR $\left(\mathrm{CD}_{3} \mathrm{CN}\right): \delta=6.46\left(\mathrm{~d}, 1 \mathrm{H}, \mathrm{C}_{2}\right.$-thiazole), $7.13-7.67(\mathrm{~m}$, $10 \mathrm{H}, \mathrm{Ar}-\mathrm{H}), 8.11$ (d, 1H, C $\mathrm{C}_{3}$-thiazole). Anal. Calcd for $\mathrm{C}_{17} \mathrm{H}_{12} \mathrm{Cl}_{6} \mathrm{~N}_{3} \mathrm{SSb}$ (624.84): C, 32.68; H, 1.94; N, 6.72. Found: C, 32.51; H, 2.15; N, 6.84 .

4-(Methylthio)-2-phenyl-thiazolo[3,2-a][1,3,5]triazi n-5-ylium hexachloroantimonate (6b): From trichlorophenylmethane $(1.96 \mathrm{~g}, 10 \mathrm{mmol})$ and methyl thiocyanate $(0.73 \mathrm{~g}, 10 \mathrm{mmol})$ as described for $\mathbf{1}$. After cooling to $+23^{\circ} \mathrm{C}$, N-trimethylsilyl-aminothiazol [25] $(1.72 \mathrm{~g}, 10$ $\mathrm{mmol}$ ) in absolute dichloroethane was added to the mixture. The reaction mixture was boiled under reflux for 3 hours, and then cooled to room temperature. The solvent was removed under reduced pressure and the residue recrystallized from dichloroethane to afford yellow powder. Yield: $(3.4$ g, $57.2 \%)$, m.p. $216^{\circ} \mathrm{C}-218^{\circ} \mathrm{C}$. IR (KBr): $v=1562,1593,3109 \mathrm{~cm}^{-1} .{ }^{1} \mathrm{H}$ NMR (DMSO-d $\left.\mathrm{d}_{6}\right): \delta=$ $2.76\left(\mathrm{~s}, 3 \mathrm{H}, \mathrm{CH}_{3}\right), 7.42$ (d, $1 \mathrm{H}, \mathrm{C}_{2}$-thiazole), $7.60-7.70$ (m, 5H, Ar-H), 8.05 (d, 1H, C $\mathrm{C}_{3}$-thiazole). Anal. Calcd for $\mathrm{C}_{12} \mathrm{H}_{10} \mathrm{Cl}_{6} \mathrm{~N}_{3} \mathrm{~S}_{2} \mathrm{Sb}$ (594.84): C, 24.23; H, 1.69; N, 7.06. Found: C, 24.45; H, 1.49; N, 6.87 .

4,6-Diphenyl-1-(substituted)-1H-[1,3,5]triazine-2-thiones (7a,b): From trichlorophenylmethane (1.96 g, 10 $\mathrm{mmol})$ and benzonitrile $(1.03 \mathrm{~g}, 10 \mathrm{mmol})$ as described for $\mathbf{1}$, and cooling to $+23^{\circ} \mathrm{C}$, a suspension of a suspension of thiosemicarbazone derivatives $(10 \mathrm{mmol})$ in 1,2 -dichloroethane was added to the mixture. The reaction mixture was boiled under reflux for 5 hours. The solvent was removed under reduced pressure and the hexachloroantimate residue was stirred in $\mathrm{CH}_{2} \mathrm{Cl}_{2}(15 \mathrm{~mL})$ with $\mathrm{Na}_{2} \mathrm{CO}_{3}(10.60 \mathrm{~g}, 100 \mathrm{mmol})$ in water $(30 \mathrm{~mL}) 3$ hours. The organic layer was separated, the aqueous solution extracted with $\mathrm{CH}_{2} \mathrm{Cl}_{2}(2 \times 50 \mathrm{~mL})$ and the combined organic extracts are dried $\left(\mathrm{Na}_{2} \mathrm{SO}_{4}\right)$. Evaporation of the solvent and crystallization of the residue afforded the corresponding triazine derivatives $7 \mathbf{a}, \mathbf{b}$.

1-[(4-Methylbenzylidene)-amino]-4,6-diphenyl-1H-[ 1,3,5]triazine-2-thione (7a): from 1-(4-tolylmethylene) thiosemicarbazide: The residue crystallized from chloroform/petroleum ether to afford red powder. Yield: (1.00 g, $52.6 \%$ ), m.p. $189^{\circ} \mathrm{C}-191^{\circ} \mathrm{C}$. IR (KBr): $v=1616 \mathrm{~cm}^{-1}$ $(\mathrm{C}=\mathrm{N}) .{ }^{1} \mathrm{H}$ NMR $\left(\mathrm{DMSO}-\mathrm{d}_{6}\right): \delta=2.39\left(\mathrm{~s}, 3 \mathrm{H}, \mathrm{CH}_{3}\right), 7.49$ - $7.82(\mathrm{~m}, 14 \mathrm{H}, \mathrm{Ar}-\mathrm{H}), 8.11(\mathrm{~s}, 1 \mathrm{H}, \mathrm{CH}=\mathrm{N}) . \mathrm{MS} \mathrm{m} / \mathrm{z}=$ 381 (2.2\%), 221 (86.3), 118 (100\%), 77 (30.8\%). Anal. Calcd for $\mathrm{C}_{23} \mathrm{H}_{18} \mathrm{~N}_{4} \mathrm{~S}$ (382.48): C, 72.22; H, 4.74; N,
14.65. Found: C, 72.40; H, 4.68; N, 14.52 .

4,6-Diphenyl-1-[(quinolin-2-ylmethylene)-amino]-1 $H$-[1,3,5]triazin-2-thione (7b): from 1-(quinolin-2ylmethylene) thiosemicarbazide, The residue crystallized from petroleum ether to afford brown powder. Yield: (1.2 g, $57.4 \%$ ), m.p. $154^{\circ} \mathrm{C}-157^{\circ} \mathrm{C}$ (dec.). IR (KBr): $v=$ $1597 \mathrm{~cm}^{-1}(\mathrm{C}=\mathrm{N}) .{ }^{1} \mathrm{H}$ NMR $\left(\right.$ DMSO-d $\left.{ }_{6}\right): \delta=7.05-7.85$ $(\mathrm{m}, 16 \mathrm{H}, \mathrm{Ar}-\mathrm{H}), 8.22(\mathrm{~s}, 1 \mathrm{H}, \mathrm{CH}=\mathrm{N}) . \mathrm{MS} \mathrm{m} / \mathrm{z}=419$ (16.8\%), 250 (24.9), 118 (58.6\%), 147 (100\%), 121 (87.4\%), 77 (64.1\%). Anal. Calcd for $\mathrm{C}_{25} \mathrm{H}_{17} \mathrm{~N}_{5} \mathrm{~S}$ (419.50): C, 71.58; H, 4.08; N, 16.69. Found: C, 71.77; H, 4.26; N, 16.65 .

5-(Methylthio)-3-phenyl-1H-[1,2,4]triazole-4-iumhexachloroantimonate (8): From trichlorophenylmethane $(1.96 \mathrm{~g}, 10 \mathrm{mmol})$ and methyl thiocyanate $(0.73 \mathrm{~g}, 10$ mmol) described for $\mathbf{1}$. After cooling to $+23^{\circ} \mathrm{C}$, a suspension of benzohydrazone $(1.36 \mathrm{~g}, 10 \mathrm{mmol})$ in 1,2-dichloroethane was added dropwise to the mixture and stirring was continued for 2 hours at room temperature. The solvent was removed under reduced pressure and the residue recrystallized from dichloromethane to afford yellow powder. Yield: $(3.2 \mathrm{~g}, 60.7 \%)$, m.p. $232^{\circ} \mathrm{C}-$ $235^{\circ} \mathrm{C}$. IR $(\mathrm{KBr}): v=1608(\mathrm{C}=\mathrm{N}), 3329 \mathrm{~cm}^{-1}(\mathrm{NH}) .{ }^{1} \mathrm{H}$ NMR (DMSO-d $\left.{ }_{6}\right): \delta=2.61\left(\mathrm{~s}, 3 \mathrm{H}, \mathrm{CH}_{3}\right), 7.49-7.97(\mathrm{~m}$, $5 \mathrm{H}, \mathrm{Ar}-\mathrm{H}), 11.59$ (s, 1H, NH). Anal. Calcd for

$\mathrm{C}_{9} \mathrm{H}_{10} \mathrm{Cl}_{6} \mathrm{~N}_{3} \mathrm{SSb}$ (526.74): C, 20.52; H, 1.91; N, 7.98. Found: C, 20.23; H, 1.75; N, 7.75.

1,5-Diphenyl-3-(3,4,5-trimethoxyphenyl)-1H-[1,2,4] triazole (9): From trichlorophenylmethane (1.96 g, 10 $\mathrm{mmol})$ and 3,4,5-trimethoxybenzonitile $(1.93 \mathrm{~g}, 10 \mathrm{mmol})$ as described for 1 . After cooling to $+23^{\circ} \mathrm{C}$, phenyl hydrazine $(1.08,10 \mathrm{mmol})$ in 1,2-dichloroethane was added to the mixture. The reaction mixture was boiled under reflux for 1 hour. The solvent was removed under reduced pressure and the residue in $\mathrm{CH}_{2} \mathrm{Cl}_{2}(15 \mathrm{~mL})$ treated directly with $\mathrm{Na}_{2} \mathrm{CO}_{3}(5.30 \mathrm{~g}, 50 \mathrm{mmol})$ in water $(30 \mathrm{~mL})$ and stirred for 3 hours. The organic layer was separated, the aqueous solution extracted with $\mathrm{CH}_{2} \mathrm{Cl}_{2}(2 \times 50 \mathrm{~mL})$ and the combined organic extracts are dried $\left(\mathrm{Na}_{2} \mathrm{SO}_{4}\right)$. The solvent was evaporated the residue was crystallized from dichloromethane $/ n$-pentane to afford reddish brown powder. Yield: $(2.6 \mathrm{~g}, 67.18 \%)$, m.p. decom. $190^{\circ} \mathrm{C}$. IR $(\mathrm{KBr}): v=1261(\mathrm{C}=\mathrm{N}), 2962 \mathrm{~cm}^{-1}(\mathrm{C}-\mathrm{H}) .{ }^{1} \mathrm{H}$ NMR $\left(\mathrm{DMSO}_{\mathrm{d}}\right): \delta=3.81\left(\mathrm{~s}, 9 \mathrm{H}, \mathrm{OCH}_{3}\right), 6.85-6.90(\mathrm{~s}, 2 \mathrm{H}$, Ar-H), 7.25 - 7.58 (m, 10H, Ar-H). MS m/z = 387 (3.4\%), 338 (5.4\%), 312 (100\%), 284 (48\%), 127 (18.2\%), 77 (83.8\%). Anal. Calcd for $\mathrm{C}_{23} \mathrm{H}_{21} \mathrm{~N}_{3} \mathrm{O}_{3}$ (387.43): C, 71.30; H, 5.46; N, 10.85. Found: C, 71.08; H, 5.29; N, 11.02.

6-Methyl-4-(methylthio)-2-phenyl-benzo[e][1,3]oxa zin-1-ylium hexachloroantimonate (10): From trichlorophenylmethane $(1.96 \mathrm{~g}, 10 \mathrm{mmol})$ and methyl thiocyanate $(0.73 \mathrm{~g}, 10 \mathrm{mmol})$ as described for $\mathbf{1}$. After cooling to $+23^{\circ} \mathrm{C}$, a suspension of $p$-cresol $(1.19 \mathrm{~g}, 11 \mathrm{mmol})$ in 1,2-dichloroethane was added to the mixture. The reac- 
tion mixture was boiled under reflux for 1 hour. After cooling to room temperature, the solvent was removed under reduced pressure and the residue recrystallized from dichloromethane to afford orange crystal. Yield: $(3.2 \mathrm{~g}$, $53.10 \%)$, m.p. $245^{\circ} \mathrm{C}-247^{\circ} \mathrm{C}$. IR $(\mathrm{KBr}): v=1593(\mathrm{C}=\mathrm{N})$, $2943 \mathrm{~cm}^{-1}(\mathrm{C}-\mathrm{H}) .{ }^{1} \mathrm{H}$ NMR $\left(\mathrm{CD}_{3} \mathrm{CN}\right): \delta=2.45(\mathrm{~s}, 3 \mathrm{H}$, $\left.\mathrm{CH}_{3}\right), 2.69$ (s, 3H, $\left.\mathrm{SCH}_{3}\right), 7.41-8.18(\mathrm{~m}, 8 \mathrm{H}, \mathrm{Ar}-\mathrm{H}) .{ }^{13} \mathrm{C}$ NMR $\left(\mathrm{CD}_{3} \mathrm{CN}\right): \delta=16.16,19.82,123.92,124.23$, 128.50, 129.09 (2C), 130.19, 130.31(2C), 134.63, 136.53, 137.11, 137.76, 145.23, 164.00. Anal. Calcd for

$\mathrm{C}_{16} \mathrm{H}_{14} \mathrm{Cl}_{6} \mathrm{NOSSb}$ (602.83): C, 31.88; H, 2.34; N, 2.32. Found: C, 31.71; H, 2.43; N, 2.41 .

3-Methyl-pyrazolo[4,3-e][1,3]oxazin-7-ylium hexachloroantimonates (11a,b): From trichlorophenylmethane $(1.96 \mathrm{~g}, 10 \mathrm{mmol})$ and benzonitrile or methyl thiocyanate (10 mmol) as described for 1 . After cooling to $+23^{\circ} \mathrm{C}$, a suspension of 3-methyl-1-phenyl-5-pyrazolone (1.74 g, 10 $\mathrm{mmol}$ ) in 1,2-dichloroethane was added to the mixture. The reaction mixture boiled under reflux for 4 hours. After cooling to room temperature, the solvent was removed under reduced pressure and the residue recrystallized from dichloromethane to afford orange crystals from $11 \mathrm{a}$ or $11 \mathrm{~b}$.

11a: Yield: $(4.6 \mathrm{~g}, 76.4 \%)$, m.p. $264^{\circ} \mathrm{C}-268^{\circ} \mathrm{C}$. IR $(\mathrm{KBr}): v=1527,1558,1581,1612,3064 \mathrm{~cm}^{-1} .{ }^{1} \mathrm{H}$ NMR $\left(\mathrm{DMSO}_{6}\right): \delta=2.29\left(\mathrm{~s}, 3 \mathrm{H}, \mathrm{CH}_{3}\right), 7.41-8.10(\mathrm{~m}, 15 \mathrm{H}$, Ar-H). Anal. Calcd for $\mathrm{C}_{24} \mathrm{H}_{18} \mathrm{Cl}_{6} \mathrm{~N}_{3} \mathrm{OSb}$ (698.90): C, 41.24; H, 2.60; N, 6.01. Found: C, 41.37; H, 2.76; N, 6.13 .

11b: Yield: $\left(3.8 \mathrm{~g}, 56.8 \%\right.$ ), m.p. $235^{\circ} \mathrm{C}-238^{\circ} \mathrm{C}$ (dec.). IR (KBr): $v=1527,1558,1595,3077 \mathrm{~cm}^{-1} .{ }^{1} \mathrm{H}$ NMR (DMSO-d $\left.)_{6}\right): \delta=2.37\left(\mathrm{~s}, 3 \mathrm{H}, \mathrm{CH}_{3}\right), 2.47\left(\mathrm{~s}, 3 \mathrm{H}, \mathrm{SCH}_{3}\right)$, 7.40 - 8.06 (m, 10H, Ar-H). Anal. Calcd for

$\mathrm{C}_{19} \mathrm{H}_{16} \mathrm{Cl}_{6} \mathrm{~N}_{3} \mathrm{OSSb}$ (668.89): C, 34.12; H, 2.41; N, 6.28. Found: C, 34.33; H, 2.33; N, 6.14.

4-Chloro-6-(4-chlorophenyl)-5-cyano-2-phenyl-pyri midin-1-ium hexachloroantimonate (12): From trichlorophenylmethane $(1.96 \mathrm{~g}, 10 \mathrm{mmol})$ and $p$-chlorobenzonitrile (1.38 g, $10 \mathrm{mmol})$ as described for $\mathbf{1}$. After cooling to $+23^{\circ} \mathrm{C}$, a suspension malononitrile $(0.66 \mathrm{~g}, 10 \mathrm{mmol})$ in 1,2-dichloroethane was added to the mixture. The reaction mixture was boiled under reflux for 6 hours. After cooling to room temperature, the solvent was removed under reduced pressure and the residue recrystallized from dichloromethane to afford light brown powder. Yield: (5.0 g, $82.9 \%)$, m.p. $200^{\circ} \mathrm{C}-204^{\circ} \mathrm{C}$. IR (KBr): $v=1492$, 1550, $1597(\mathrm{C}=\mathrm{N}), 2264(\mathrm{C} \equiv \mathrm{N}), 3209 \mathrm{~cm}^{-1}(\mathrm{NH}) .{ }^{1} \mathrm{H}$ NMR $\left(\mathrm{CDCl}_{3}\right): \delta=7.51-8.54(\mathrm{~m}, 9 \mathrm{H}, \mathrm{Ar}-\mathrm{H}), 10.33(\mathrm{~s}$, $1 \mathrm{H}, \mathrm{NH}$ ). Anal. Calcd for $\mathrm{C}_{17} \mathrm{H}_{10} \mathrm{Cl}_{8} \mathrm{~N}_{3} \mathrm{Sb}$ (661.66): C, 30.86; H, 1.52; N, 6.35. Found: C, 30.72; H, 1.61; N, 6.43 .

4-Chloro-6-(4-chlorophenyl)-2-phenylpyrimidine-5carbonitrile (13): A mixture of the hexachloroantimate 12 (3.0 g, $5 \mathrm{mmol})$ in $\mathrm{CH}_{2} \mathrm{Cl}_{2}(15 \mathrm{~mL})$ and $\mathrm{Na}_{2} \mathrm{CO}_{3}(5.30$ $\mathrm{g}, 50 \mathrm{mmol})$ in water $(30 \mathrm{~mL})$ is stirred for $3 \mathrm{hr}$. the organic layer is separated, the aqueous solution extracted with $\mathrm{CH}_{2} \mathrm{Cl}_{2}(2 \times 50 \mathrm{~mL})$ and the combined organic extracts are dried $\left(\mathrm{Na}_{2} \mathrm{SO}_{4}\right)$. The solvent was evaporated and the residue was crystallized from dichloromethane to afford brownish white powder. Yield: (1.2 g, 57.7\%), m.p. $178^{\circ} \mathrm{C}-180^{\circ} \mathrm{C}$ (dec.). IR $(\mathrm{KBr}): v=1595(\mathrm{C}=\mathrm{N})$, $2229(\mathrm{C} \equiv \mathrm{N}) \mathrm{cm}^{-1}$. Anal. Calcd for $\mathrm{C}_{17} \mathrm{H}_{9} \mathrm{Cl}_{2} \mathrm{~N}_{3}(326.18)$ : C, 62.60; H, 2.78; N, 12.88. Found: C, 62.73; H, 2.81; N, 12.93.

4-(4-Chlorophenyl)-6-phenyl-1H-pyrazolo[3,4-d]py rimidin-3-ylamine (14): A mixture of $\mathbf{1 3}$ (4.19 g, 10 mmol) in ethanol $(25 \mathrm{~mL})$ and $20 \mathrm{mmol}$ of hydrazine hydrate in ethanol $(30 \mathrm{~mL})$ was refluxed for 4 hours. The solid product that separated on cooling was filtered off, dried, and recrystallized from ethanol to afford pale green powder. Yield: $(2.3 \mathrm{~g}, 71.8 \%)$, m.p. over $260^{\circ} \mathrm{C}$. IR $(\mathrm{KBr}): v=1589(\mathrm{C}=\mathrm{N}), 3197,3352, \mathrm{~cm}^{-1}\left(\mathrm{NH}_{2}\right) .{ }^{1} \mathrm{H}$ NMR (DMSO-d $\left.\mathrm{d}_{6}\right): \delta=7.32-7.87(\mathrm{~m}, 9 \mathrm{H}, \mathrm{Ar}-\mathrm{H}), 8.80$ (s, $1 \mathrm{H}, \mathrm{NH}), 10.22\left(\mathrm{~s}, 2 \mathrm{H}, \mathrm{NH}_{2}\right), 12.8(\mathrm{~s}, 1 \mathrm{H}, \mathrm{NH})$. Anal. Calcd for $\mathrm{C}_{17} \mathrm{H}_{12} \mathrm{ClN}_{5}$ (321.76): C, 63.46; H, 3.76; N, 21.77. Found: C, 63.25; H, 3.89; N, 21.65.

N-(4-(4-Chlorophenyl)-6-phenyl-1H-pyrazolo[3,4-d Jpyrimidin-3-yl)benzothioamide (15): A mixture of 14 $(3.21 \mathrm{~g}, 10 \mathrm{mmol})$ and phenyl isothiocyanate $(1.35 \mathrm{~g}, 10$ $\mathrm{mmol})$ in dry pyridine $(20 \mathrm{~mL})$ was heated under reflux for 4 hours. After cooling, the reaction mixture was poured into cold water $(20 \mathrm{~mL})$ with stirring. The product was filtered off, dried, and recrystallized from ethanol to afford light brown powder. Yield: $(2.1 \mathrm{~g}, 47.5 \%)$, m.p. $160^{\circ} \mathrm{C}-164^{\circ} \mathrm{C}$. IR (KBr): $v=3028(\mathrm{C}=\mathrm{N}), 3132$, $3197,3352 \mathrm{~cm}^{-1}$ (NH groups). ${ }^{1} \mathrm{H}$ NMR (DMSO-d $\left.\mathrm{d}_{6}\right): \delta=$ $7.51-8.51$ (m, 14H, Ar-H), 8.70 (s, 1H, NH), 8.82 (s, 1H, $\mathrm{NH}), 12.8(\mathrm{~s}, 1 \mathrm{H}, \mathrm{NH})$. Anal. Calcd for $\mathrm{C}_{24} \mathrm{H}_{17} \mathrm{ClN}_{6} \mathrm{~S}$ (456.95): C, 63.08; H, 3.75; N, 18.39. Found: C, C, 63.02; H, 3.78; N, 18.44 .

N-(Arylidene)-4-(4-chlorophenyl)-6-phenyl-1H-pyr azolo[3,4-d]pyrimidin-3-ylamine (16a,b): A mixture of 14 (3.21 g, $10 \mathrm{mmol})$ and $p$-anisaldehyde or $p$-toulaldehyde $(10 \mathrm{mmol})$ was refluxed for 4 hours in glacial acetic acid $(25 \mathrm{~mL})$ containing sodium acetate $(0.41 \mathrm{~g}, 5 \mathrm{mmol})$. The reaction mixture was allowed to cool at room temperature, and then reaction mixture was poured into cold water. The solid product was collected by filtration, dried and recrystallized from ethanol to afford the corresponding arylidene derivatives $\mathbf{1 6}$ as orange powder.

16a: Yield: $(2.0$ g, $45.5 \%)$, m.p. $195^{\circ} \mathrm{C}-197^{\circ} \mathrm{C}$. IR $(\mathrm{KBr}): v=1516,1566,1593,2916,3032,3147,3205$ $\mathrm{cm}^{-1} .{ }^{1} \mathrm{H}$ NMR (DMSO-d $): \delta=3.85\left(\mathrm{~s}, 3 \mathrm{H}, \mathrm{OCH}_{3}\right), 7.08$ - 8.18 (m, 14H, Ar-H), 12.84 (s, 1H, NH). MS m/z = 439 (79.7\%), m + 2441 (43.2\%), 332 (100\%), $219(20.9 \%)$, 169 (16.2\%), 77 (34.5\%). Anal. Calcd for $\mathrm{C}_{25} \mathrm{H}_{18} \mathrm{ClN}_{5} \mathrm{O}$ (439.90): C, 68.26; H, 4.12; N, 15.92. Found: C, 68.373; H, 4.14; N, 15.87 . 
16b: Yield: $(3.1 \mathrm{~g}, 73.1 \%)$, m.p. $187^{\circ} \mathrm{C}-190^{\circ} \mathrm{C}$. IR $(\mathrm{KBr}): v=1498,1593,2885,3028,3105,3143,3209$ $\mathrm{cm}^{-1} .{ }^{1} \mathrm{H}$ NMR (DMSO-d $\left.\mathrm{d}_{6}\right): \delta=3.30\left(\mathrm{~s}, 3 \mathrm{H}, \mathrm{CH}_{3}\right), 7.24-$ 7.97 (m, 14H, Ar-H), 13.97 (s, 1H, NH). MS m/z = 423 (71.5\%), m + 2425 (26.2\%), $332(100 \%), 212(12.7 \%)$, 138 (13.1\%), 104 (25.7\%), 77 (31.5\%). Anal. Calcd for $\mathrm{C}_{25} \mathrm{H}_{18} \mathrm{ClN}_{5}$ (423.90): C, 70.84; H, 4.28; N, 16.52. Found: C, $70.91 ; \mathrm{H}, 4.31 ; \mathrm{N}, 16.48$.

\subsection{Antimicrobial Activity}

The free base Compounds $(3,5,7 a, 7 b, 9,13,14,15$, 16a and 16b) were screened for their in-vitro antibacterial activity against garm $(+)$ bacteria; [Staphlococcus aurous, Staphylococcus epidormidis, Stroptococcus faecalis] and gram(-) bacteria; [Escherichi coli, Klebsiella pneumonia, Proteus mirabilis, Pseudomonus aeuroginosa] employing well-diffusion method at the concentration of $100 \mu \mathrm{g} / \mathrm{mL}$ and $150 \mu \mathrm{g} / \mathrm{mL}$ in Müller-Hinton agar media and also for in-vitro antifungal activity against Candida albicans and Saccharomyces cervisiae by welldiffusion method at the concentration of $100 \mu \mathrm{g} / \mathrm{mL}$ and $150 \mu \mathrm{g} / \mathrm{mL}$ using sabouraud-dextrose agar. DMF was used as a solvent control for antimicrobial activity. (CN10) Gentamicin, (CTX30) Cefotaxim, (FOX30) Cefoxitix, (E15) Erythromycin, (TE30) Tetracyclin, (MXF5) Moxifloxacin, (VA30) Vancomycin and (CIP5) Ciprofloxacin were used as reference antibacterial drugs where (CLT10) Clotrimazole and (FCA25) Fluconazole as reference antifungal drugs. The area of inhibition of zone measured in $\mathrm{mm}$. The results are listed in Table $\mathbf{1}$.

Table 1. Concentration in $\mu \mathrm{g} / \mathrm{mL}$, Diameter of inhibition zone (mm).

\begin{tabular}{|c|c|c|c|c|c|c|c|c|c|c|c|c|c|c|c|c|c|c|}
\hline \multirow{3}{*}{$\begin{array}{l}\text { Comp. } \\
\text { Conc. } \\
\mu \mathrm{g} / \mathrm{mL}\end{array}$} & \multicolumn{6}{|c|}{$\operatorname{Gram}(+)$ bacteria } & \multicolumn{8}{|c|}{$\operatorname{Gram}(-)$ bacteria } & \multicolumn{4}{|c|}{ Fungi } \\
\hline & \multicolumn{2}{|c|}{ S. aurous } & \multicolumn{2}{|c|}{ S. epidormidis } & \multicolumn{2}{|c|}{ S. faecalis } & \multicolumn{2}{|c|}{ K. pneumonia } & \multicolumn{2}{|c|}{ P. mirabilis } & \multicolumn{2}{|c|}{ P. aeuroginosa } & \multicolumn{2}{|c|}{ E. coli } & \multicolumn{2}{|c|}{ C. albicans } & \multicolumn{2}{|c|}{ S. cervisiae } \\
\hline & 100 & 150 & 100 & 150 & 100 & 150 & 100 & 150 & 100 & 150 & 100 & 150 & 100 & 150 & 100 & 150 & 100 & 150 \\
\hline 3 & 6 & 13 & 11 & 10 & 14 & 14 & 15 & 16 & 14 & 15 & 8 & 14 & 15 & 15 & 6 & 13 & 6 & 20 \\
\hline 5 & 6 & 6 & 6 & 6 & 6 & 6 & 15 & 15 & 6 & 13 & 11 & 22 & 16 & 16 & 14 & 16 & 18 & 20 \\
\hline $7 a$ & 6 & 12 & 9 & 12 & 6 & 8 & 12 & 14 & 10 & 13 & 10 & 13 & 13 & 14 & 11 & 14 & 18 & 18 \\
\hline $7 b$ & 6 & 14 & 10 & 12 & 8 & 8 & 15 & 15 & 13 & 14 & 9 & 17 & 14 & 14 & 12 & 15 & 18 & 20 \\
\hline 9 & 22 & 27 & 16 & 16 & 16 & 20 & 18 & 20 & 15 & 16 & 20 & 22 & 15 & 15 & 8 & 12 & 18 & 20 \\
\hline 13 & 7 & 8 & 6 & 6 & 6 & 6 & 15 & 15 & 6 & 15 & 10 & 14 & 6 & 16 & 12 & 12 & 19 & 19 \\
\hline 14 & 8 & 10 & 7 & 9 & 6 & 6 & 11 & 14 & 8 & 12 & 8 & 12 & 12 & 14 & 10 & 12 & 17 & 18 \\
\hline 15 & 12 & 13 & 6 & 6 & 6 & 6 & 14 & 15 & 6 & 15 & 8 & 12 & 6 & 16 & 12 & 15 & 18 & 18 \\
\hline $16 a$ & 6 & 6 & 9 & 14 & 6 & 6 & 6 & 6 & 6 & 6 & 6 & 12 & 6 & 6 & 6 & 6 & 6 & 18 \\
\hline $16 b$ & 6 & 10 & 6 & 6 & 6 & 6 & 15 & 19 & 6 & 14 & 12 & 15 & 15 & 15 & 10 & 13 & 17 & 19 \\
\hline DMF & \multicolumn{2}{|c|}{ Nil } & \multicolumn{2}{|c|}{ Nil } & \multicolumn{2}{|c|}{ Nil } & \multicolumn{2}{|c|}{ Nil } & \multicolumn{2}{|c|}{ Nil } & \multicolumn{2}{|c|}{ Nil } & \multicolumn{2}{|c|}{ Nil } & \multicolumn{2}{|c|}{ Nil } & \multicolumn{2}{|c|}{ Nil } \\
\hline CN10 & \multicolumn{2}{|c|}{17} & \multicolumn{2}{|c|}{19} & \multicolumn{2}{|c|}{6} & \multicolumn{2}{|c|}{13} & \multicolumn{2}{|c|}{6} & \multicolumn{2}{|c|}{15} & 1 & & & & & \\
\hline CTX30 & & 8 & S & & 6 & & 1 & & 9 & & s & & 2 & & & & & \\
\hline FOX30 & & 0 & 1 & & 6 & & 1 & & 2 & & ( & & 2 & & & & & \\
\hline E15 & & 8 & $\varepsilon$ & & 6 & & 6 & & 6 & & 1 & & & & & & & \\
\hline TE30 & & 9 & 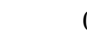 & & 6 & & 1 & & 8 & & $\varepsilon$ & & 1 & & & & & \\
\hline MXF5 & & 9 & 2 & & 22 & & 9 & & 6 & & 1 & & 1 & & & & & \\
\hline VA30 & & 3 & 1 & & 8 & & 6 & & 6 & & 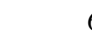 & & & & & & & \\
\hline CIP5 & & 5 & 2 & & 21 & & 2 & & 1 & & 3 & & 2 & & & & & \\
\hline CLT10 & & & & & & & & & & & & & & & & & & 22 \\
\hline FCA25 & & & & & & & & & & & & & & & & & & 10 \\
\hline
\end{tabular}




\section{RESULTS}

Substituted 2-azoniaallene salts 1 with stabilizing groups $\mathrm{R}^{1}, \mathrm{R}^{2}$ have found considerable preparative application. Azoniaallene salts are strong bifunctional electrophiles, which should undergo cyclization reactions with molecules with two nucleophilic centers. Here we repot such cyclizations. 2-Azoniaallene salts are versatile building blocks in furnishes many series of heterocyclic compounds, as triazine derivatives (Scheme 1). $p$-Tolyl urea was reacted with symmetric and asymmetric 2-azoniaallene salts $\mathbf{1}$ in dichloroethane to afford the corresponding oxotriazinium salts 2a,b. The free base triazine compound 3 was achieved by treatment of hexachloroantimonate salt 2a with $\mathrm{Na}_{2} \mathrm{CO}_{3}$ solution. The chemical structure of $\mathbf{2}$ and 3 were secured by their elemental analyses and spectral data. The IR spectrum of 2a displayed bands at $\mathrm{NH}$ band at 3282 and $1739 \mathrm{~cm}^{-1}$ due to $\mathrm{NH}$ and $\mathrm{C}=\mathrm{O}$ groups, respectively. The ${ }^{1} \mathrm{H}$ NMR spectrum of 2a showed singlet signal at $\delta=2.26 \mathrm{ppm}$ for the methyl protons, multiplet in the range $\delta=7.27-8.4$ for the aromatic protons and singlet at $\delta=11.33$ for the $(\mathrm{NH})$ proton. Under similar conditions, phenyl thiourea reacted with 2-azoniaallene salt $\mathbf{1}$ to furnish the thiotriazinium salt 4. The IR spectrum of this salt showed a sharp and intensive peak for $\mathrm{NH}$ at $3240 \mathrm{~cm}^{-1}$. The ${ }^{1} \mathrm{H}$ NMR showed multiplet signal of aromatic protons and singlet signal a of $(\mathrm{NH})$ proton at $7.53-8.57$ and 10.82, respectively. The free thiotriazine compound 5 was obtained by treatment the corresponding salt $\mathbf{4}$ with solution of $\mathrm{Na}_{2} \mathrm{CO}_{3}$. The mass spectrum of compound $\mathbf{5}$ showed the molecular ion peak at $\mathrm{m} / \mathrm{z}=411$ corresponding to the formula $\mathrm{C}_{21} \mathrm{H}_{13} \mathrm{Cl}_{2} \mathrm{~N}_{3} \mathrm{~S}$. Aminothiazole with a good leaving group was reacted with 2-azoniaallene salts 1 to afford the bicyclic thiazolo[3,2-a][1,3,5]triazinium salts 6a,b which seems to represent a new ring system. The NMR spectrum of 6a showed a doublet signal at $\delta=$ 6.46 for $\mathrm{C}_{2}$-thiazole proton, a multiplet signal in the region $\delta=7.13-7.67$ for the aromatic protons and a doublet signal at $\delta=8.11$ for $\mathrm{C}_{3}$-thiazole proton. Moreover, the reaction of thiosemicarbazone derivatives with symmetric azoniaallene salt $\mathbf{1}\left(\mathrm{R}^{1}=\mathrm{R}^{2}=\mathrm{Ph}\right)$ afforded the corresponding triazinium salts, which underwent neutralization by $\mathrm{Na}_{2} \mathrm{CO}_{3}$ solution furnished the corresponding triazine derivatives 7a,b (Scheme 1).<smiles>[R]c1nc([R])[n+]2ccsc2n1</smiles>

6a, $\mathbf{R}^{\mathbf{1}}=\mathbf{R}^{2}=\mathbf{P h}$

6b, $R^{1}=P h, R^{2}=S M e$

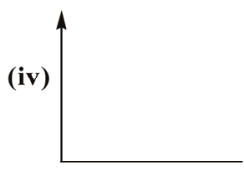

(v)<smiles>[3H]CC</smiles><smiles>[R]C=Nn1c([R])nc([R])nc1=S</smiles>

$7 \mathrm{a}, \mathbf{R}^{1}=\mathbf{R}^{2}=\mathrm{Ph}, \mathrm{R}^{3}=p-\mathrm{CH}_{3} \mathrm{C}_{6} \mathrm{H}_{4}$

$7 b, \mathbf{R}^{1}=\mathbf{R}^{\mathbf{2}}=\mathbf{P h}, \mathbf{R}^{\mathbf{3}}=\mathbf{2}$-Quinolinyl

$2 \mathbf{b}, \mathbf{R}^{1}=\mathbf{P h}, \mathbf{R}^{2}=\mathrm{SMe}$

(ii)<smiles>[R]c1nc([R])n([Ga])c(=O)n1</smiles>

3, $\mathbf{R}^{1}=\mathbf{R}^{2}=\mathbf{P h}$

$\mathrm{Ar}=4-\mathrm{MeC}_{6} \mathrm{H}_{4}$

(i)<smiles>[R]C(Cl)=[N+]([R])Cl</smiles>

$\stackrel{\text { (iii) }}{\longrightarrow}$

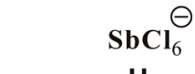<smiles></smiles>

$\mathrm{R}^{1}=\mathrm{R}^{2}=p-\mathrm{ClC}_{6} \mathrm{H}_{4}$<smiles>[R]c1nc([R])n(-c2ccccc2)c(=S)n1</smiles>

$5 \quad\left(R^{1}=R^{2}=p-\mathrm{ClC}_{6} \mathrm{H}_{4}\right)$

(i) 4- $\mathrm{MeC}_{6} \mathrm{H}_{4} \mathrm{NHCONH}_{2}$, (ii) $\mathrm{Na}_{2} \mathrm{CO}_{3}$, (iii) $\mathrm{PhNHCSNH}_{2}$, (iv) N-trimethylsilyl-aminothiazole, $\mathrm{ArCH}=\mathrm{NNHCSNH}_{2}(\mathrm{Ar}=p$-tolyl, 2-quinolinyl).

Scheme 1. Synthesis of triazine derivatives 2-7. 
New derivatives of triazoles and oxazines were synthesized through cycloaddition of 2-azoniaallene salts. Triazole salt 8 was obtained in simple reaction of azoniaallene salt 1 and benzohydrazide under stirring conditions for 30 minutes and its structure was secured by the presence of $\mathrm{NH}$ band at $3329 \mathrm{~cm}^{-1}$ and disappearance the $(\mathrm{C}=\mathrm{O})$ band of benzohydrazide. The asymmetric 2-azoniaallene salt reacted with phenyl hydrazine under reflux to afford the triazole salt which failed to be crystallized so treated directly with sodium carbonate to furnish the triazole derivative $\mathbf{9}$. The mass spectrum of 9 confirmed the presence of molecular ion peak at $\mathrm{m} / \mathrm{z}=387$ for the molecular formula $\mathrm{C}_{23} \mathrm{H}_{21} \mathrm{~N}_{3} \mathrm{O}_{3}$. Cyclization of azoniaallene to afford the benzoxazine salt $\mathbf{1 0}$ was acquired when p-cresol and 2-azoniaallene salt reacted in $(1: 1)$ ratio. On the reaction of 3-methyl-1-phenyl-5-pyrazolone with azoniaallene salts, a good yield of 11a,b was obtained (Scheme 2).

Primidinium salt 12, formed under reaction of malononitrile with azoniaallene salt $\mathbf{1}$, was confirmed by IR which showed sharp band for nitrile group at $2264 \mathrm{~cm}^{-1}$, and $3209 \mathrm{~cm}^{-1}$ for NH group. Treatment of salt 12 with sodium carbonate solution afforded the corresponding free pyrimidine base $\mathbf{1 3}$ with the same sharp band for nitrile group at $2229 \mathrm{~cm}^{-1}$. The bicyclic system 3-aminopyrazolo[3,4-d]pyrimidine $\mathbf{1 4}$ was obtained by reflux 13 and hydrazine hydrate in ethanol. The chemical structure of 14 was based on its spectral data and elemental analysis. The amino group of $\mathbf{1 4}$ is highly versatile; it is highly reactive for nucleophilic addition to phenyl isothiocy- anate in hot pyridine to furnish the corresponding thiourea derivative 15. Moreover, compound 14 undergo condensation with aromatic aldehyde e.g. $p$-anisaldehyde and $p$-toulaldehyde to give the corresponding imine derivatives 16a,b (Scheme 3 ).

\section{CONCLUSION}

In the present research studies, our efforts are to synthesize some new heterocyclic compounds triazine, triazole, oxazine, and primidine based on the bifunctional electrophile azoniaallene salts. These synthesized compounds are characterized by various elemental and spectral analyses. The antimicrobial data showed that the new compounds in general have moderate to good activity compared to the reference antibacterial \& antifungal drugs. Looking at the structure activity relationship (SAR) for compounds (7a, 7b) observed that the change in chemical structure has no significant effect on growth inhibition of bacteria and fungi. Where for $(\mathbf{1 6 a}, \mathbf{1 6 b})$ there are some differences especially in the diameter of inhibition zone $(\mathrm{mm})$ of Gram(-) bacteria and fungi. Work is underway in our laboratories to increase the efficacy and specificity of the titled scaffolds as antimicrobial by structural refinements and modulation.

\section{ACKNOWLEDGEMENTS}

The authors wish to express their deep gratitude to Dr. Ehab Abdellatif, Chemistry Department, Faculty of Science, Mansoura University, Egypt, for his helpful discussion. Also Dr. Alaa El-din A. Baker,<smiles></smiles>

10, $\mathbf{R}^{1}=\mathbf{P h}, \mathbf{R}^{2}=\mathbf{S M e}$

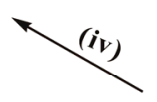

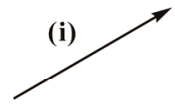

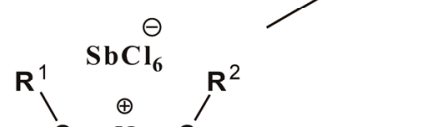

CI

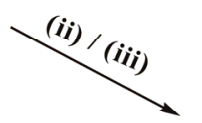

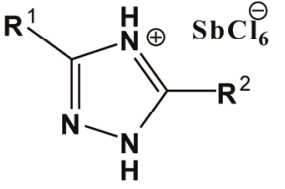

$$
8, \mathbf{R}^{1}=\mathbf{P h}, \mathbf{R}^{2}=\mathrm{SMe}
$$

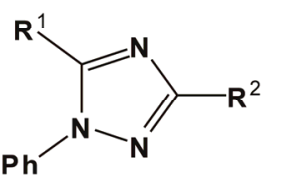

9

$\mathbf{R}^{1}=\mathbf{P h}$,

$\mathrm{R}^{2}=3,4,5-(\mathrm{MeO})_{3} \mathrm{C}_{6} \mathrm{H}_{2}$

$11 \mathrm{a}, \mathrm{K}^{1}=\mathrm{R}^{2}=\mathbf{P h}$

$11 \mathrm{~b}, \mathrm{R}^{1}=\mathbf{P h}, \mathbf{R}^{\mathbf{2}}=\mathrm{SMe}$

(i) $\mathrm{PhCONHNH}_{2}$, (ii) $\mathrm{PhNHNH}_{2}$, (iii) $\mathrm{Na}_{2} \mathrm{CO}_{3}$, (iv) $p$ - $\mathrm{MeC}_{6} \mathrm{H}_{4} \mathrm{OH}$, (v) 3-methyl-1-phenyl-5-pyrazolone.

Scheme 2. Synthesis of triazole and oxazine derivatives 8-11. 


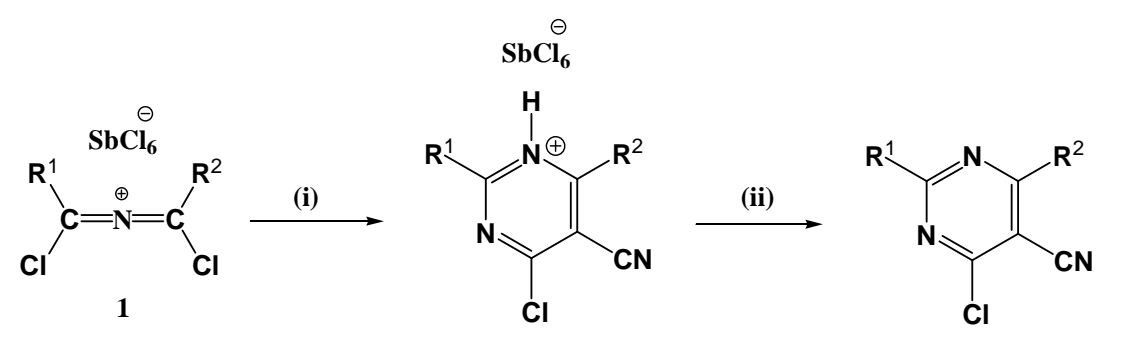

$12, \mathbf{R}^{1}=\mathrm{Ph}, \mathrm{R}^{2}=p-\mathrm{ClC}_{6} \mathrm{H}_{4}$<smiles>[R][R]c1nc([R])nc2[nH]nc(N=C[R])c12</smiles>

16a, $\mathrm{R}^{1}=\mathrm{Ph}, \mathrm{R}^{2}=\mathrm{p}-\mathrm{ClC}_{6} \mathrm{H}_{4}, \mathrm{R}^{3}=p-\mathrm{CH}_{3} \mathrm{OC}_{6} \mathrm{H}_{4}$ 16b, $\mathrm{R}^{1}=\mathrm{Ph}, \mathrm{R}^{2}=\mathrm{p}-\mathrm{ClC}_{6} \mathrm{H}_{4}, \mathrm{R}^{3}=p-\mathrm{CH}_{3} \mathrm{C}_{6} \mathrm{H}_{4}$
13, $\mathbf{R}^{1}=\mathbf{P h}, \mathbf{R}^{2}=p-\mathrm{ClC}_{6} \mathrm{H}_{4}$

(iii)<smiles>[R]c1nc([R])c2c(N)n[nH]c2n1</smiles>

$14, \mathrm{R}^{1}=\mathrm{Ph}, \mathrm{R}^{2}=p-\mathrm{ClC}_{6} \mathrm{H}_{4}$<smiles>[R]c1nc([R])c2c(NC3CCCC3)n[nH]c2n1</smiles>

15, $\mathbf{R}^{\mathbf{1}}=\mathbf{P h}, \mathbf{R}^{2}=p-\mathrm{ClC}_{6} \mathbf{H}_{4}$

(i) $\mathrm{CNCH}_{2} \mathrm{CN}$, (ii) $\mathrm{Na}_{2} \mathrm{CO}_{3}$, (iii) $\mathrm{NH}_{2} \mathrm{NH}_{2} \cdot \mathrm{H}_{2} \mathrm{O}$, (iv) PhNCS, (v) a: $p-\mathrm{MeOC}_{6} \mathrm{H}_{4} \mathrm{CHO}$, b: $p-\mathrm{MeC}_{6} \mathrm{H}_{4} \mathrm{CHO}$.

Scheme 3. Synthesis of 3-aminopyrazolo[3,4-d]pyrimidine derivatives 15-16.

Egyptian environmental affairs agency, Cairo, Egypt, for his help in antimicrobial activity.

\section{REFERENCES}

[1] Hamed, A., Jochims, J.C. and Przybylski, M. (1989) 2Azoniaallene salts from the reaction of chlorocarbenium salts with N-silylimines. Synthesis, 5, 400-402. doi:10.1055/s-1989-27267

[2] Samuel, B. and Wade, A.K. (1968) Dialkylideneammonium cation analogous to an allene. Journal of the Chemical Society, 1081.

[3] Hamed, A. (2005) Some reactions of 3-chloroisoindolium salts with nucleophiles: Access to isoindole derivatives and ellipticine analogues as potential antiviral agents. Journal of Chemical Research, 3, 54-58.

[4] Hamed, A., Müller, E., Al-Talib, M. and Jochims, J.C. (1987) On the reactions of $\alpha$-chlorocarbenium ions with ammonium thiocyanate and potassium cyanate. Synthesis, 8, 745-748. doi:10.1055/s-1987-28073

[5] Voges, A., Hamed, A., El-Badry, A.A., Ismail A.H. and Jochims, J.C. (1995) On the reaction of $\alpha$-chloro carbe- nium ions with sulfinylamines. Synthesis, 3, 253-260. doi:10.1055/s-1995-3902

[6] Peterson, P.E., Clifford, P.R. and Slame, F.J. (1970) Reactions of tetramethylenehalonium ions. Journal of the American Chemical Society, 92, 2840-2844. doi:10.1021/ja00712a039

[7] Wei, M.J., Fang, D.C. and Liu, R.Z. (2004) Theoretical studies on cycloaddition reactions between 1-aza-2-azoniaallene Cations and Isocyanates. European Journal of Organic Chemistry, 2004, 4070-4076. doi:10.1002/ejoc.200400343

[8] Padwa, A. and Pearson, W.H. (2002) Chemistry toward heterocycles and natural products. John Wily \& Sons. Inc., Hoboken, 817-899.

[9] Li, G.Y., Chen, J., Yu, W.Y., Hong, W. and Che, C.M. (2003) Stereoselective synthesis of functionalized pyrrolidines by ruthenium porphyrin-catalyzed decomposition of $\alpha$-diazo esters and cascade azomethine ylide formation/1,3-dipolar cycloaddition reactions. Organic Letters, 5, 2153-2156. doi:10.1021/ol034614v

[10] Rajaian, E., Monajjemi, M. and Gholami, M.R. (2002) $A b$ initio calculation of thermodynamicand kinetic quantities for 1,3-dipolar cycloadditions of benzonitrile oxide with 
various dipolarophiles. Journal of Chemical Research, 6, 279-281. doi:10.3184/030823402103171979

[11] Wirchun, G.W., Hitzler, G.M., Jochims, J.C. and Groth, U. (2002) On alkylidene amidosulfenyl chlorides and 1-thia2-azoniaallene salts. Helvetica Chimica Acta, 85, 26272635.

doi:10.1002/1522-2675(200209)85:9<2627::AID-HLCA 2627>3.0.CO;2-H

[12] Al-Soud, Y.A. (2008) Synthesis, structure, and in vitro anti-HIV activity of new pyrazole, 1,2,4-thiadiazole, and 1,2,4-triazole derivatives. Phophorus, Sulfur, and Silicon, 183, 2621-2636. doi:10.1080/10426500801968003

[13] Leey, H.H., Cain, B.F., Denny, J.S., Bnckleron, J.S. and Clark, G.R. (1989) Synthesis and characterization of masked aminopyrazolecarboxylic acid synthons. Journal of Organic Chemistry, 54, 428-431. doi:10.1021/jo00263a032

[14] Claramut, R.M. and Elguero, J. (1991) The chemistry of pyrazolidinones. A review. Organic Preparations and Procedures International, 23, 273-320. doi:10.1080/00304949109458208

[15] Dogan, H.N., Duran, A., Rollas, S., Sener, G., Uysal, M.K. and Gülen, D. (2002) Synthesis of new 2,5-disubstituted1,3,4-thiadiazoles and preliminary evaluation of anticonvulsant and antimicrobial activities. Bioorganic \& Medicinal Chemistry, 10, 2893-2898. doi:10.1016/S0968-0896(02)00143-8

[16] Mamolo, M.G., Vio, L. and Banfi, E. (1996) Synthesis and antimicrobial activity of some 2,5-disubstituted 1,3,4thiadiazole derivatives. Il Farmaco, 51, 71-74.

[17] Schenone, S., Brullo, C., Bruno, O., Bondavalli, F., Ranise, A., Filippelli, W., Rinaldi, B., Capuano, A. and Falcone, G. (2006) New 1,3,4-thiadiazole derivatives endowed with analgesic and anti-inflammatory activities. Bioorganic \& Medicinal Chemistry, 14, 1698.

[18] Palaska, E., Sahin, G., Kelecin, P., Durlu, N.T. and Alti- nok, G. (2002) Synthesis and anti-inflammatory activity of 1-acylthiosemicarbazides, 1,3,4-oxadiazoles, 1,3,4-thiadiazoles and 1,2,4-triazole-3-thiones. Il Farmaco, 57, 101-107. doi:10.1016/S0014-827X(01)01176-4

[19] Naito, Y., Akahoshi, F., Takeda, S., Okada, T., Kajii, M., Nishimura, H., Sugiura, M., Fukaya, C. and Kagitani, Y. (1996) Synthesis and pharmacological activity of triazole derivatives inhibiting eosinophilia. Journal of Medicinal Chemistry, 39, 3019. doi:10.1021/jm9507993

[20] Clercq, E.D. (2004) Antiviral drugs in current clinical use. Journal of Clinical Virology, 30, 115-133. doi:10.1016/j.jcv.2004.02.009

[21] Jenkins, S.M., Wadsworth, H.J., Bromidge, S., Orlek, B.S., Wyman, P.A. and Riley, G.J. (1992) Substituent variation in azabicyclic triazole- and tetrazole-based muscarinic receptor ligands. Journal of Medicinal Chemistry, 35, 2392-2406. doi:10.1021/jm00091a007

[22] Al-Soud, Y.A., Al-Dweri, M.N. and Al-Masoudi, N.A. (2004) Synthesis, antitumor and antiviral properties of some 1,2,4-triazole derivatives. Il Farmaco, 59, 775-783. doi:10.1016/i.farmac.2004.05.006

[23] El-Gazzar, A.B., Hegab, M.I. and Hassan, N.A. (2002) One-pot synthesis of $\mathrm{N}$-nucleosides via 1,3-dipolar cycloaddition of 1-aza-2-azoniaallene salts to 2,3,4,6-tetraO-acetyl- $\beta$-D-glucopyranosyl isothiocyanate. Sulfur Letters, 25, 61-71. doi:10.1080/02786110213978

[24] Hassan, N.A. (2007) Syntheses of acyclic C-glycosidic derivatives of 1,2,4-triazoles by cycloadditions of 1-aza2-azoniaallene salts to D-glucononitrile-2,3,4,5,6-pentaacetate. Journal of Heterocyclic Chemistry, 44, 933-936. doi:10.1002/jhet.5570440432

[25] Schmidt, U. and Schwochau, M. (1967) Syntheses with trimethylsilyl esters of acetoacetate and malonate. A new way to diacylmethanes and diacylacetates. Monatshefte für Chemie, 98, 1492-1511. doi:10.1007/BF00909019 